Open Access

\title{
Decomposition of the large-scale atmospheric state driving downscaling: a perspective on dynamical downscaling for regional climate study
}

\author{
Seiya Nishizawa ${ }^{*}$ (D), Sachiho A. Adachi ${ }^{1}$, Yoshiyuki Kajikawa ${ }^{1,2}$, Tsuyoshi Yamaura1, Kazuto Ando1, \\ Ryuji Yoshida ${ }^{1,2}$, Hisashi Yashiro ${ }^{1}$ and Hirofumi Tomita ${ }^{1}$
}

\begin{abstract}
In this study, we provide a perspective on dynamical downscaling that includes a comprehensive view of multiple downscaling methods and a strategy for achieving better assessment of future regional climates. A regional climate simulation is generally driven by a large-scale atmospheric state obtained by a global climate simulation. We conceptualize the large-scale state based on reconstruction by combining decomposed components of the states, such as climatology and perturbation, in different global simulations. The conceptualization provides a comprehensive view of the downscaling methods of previous studies. We propose a strategy for downscaling regional climate studies based on the concept of covering a wider range of possibilities of large-scale states to account for the uncertainty in global future predictions due to model errors. Furthermore, it also extracts the individual influences of the decomposed components on regional climate change, resulting in better understanding of the cause of the change. We demonstrate a downscaling experiment to highlight the importance of the simultaneous consideration of the individual influences of climatology and perturbation.
\end{abstract}

Keywords: Dynamical downscaling, Regional climate, Uncertainty, Model errors

\section{Introduction}

Assessment of future regional climates is of growing importance in the development of adaptation plans by policymakers. Numerical simulation is a necessary tool for investigating the issue of climate change. However, global simulations are usually too coarse to represent regional-scale atmospheric variability. In order to represent regional-scale variability, dynamical downscaling (DS) with a regional model driven by a large-scale state, i.e., initial and lateral boundary conditions generated from global simulations or reanalyses, has been widely used (e.g., Dickenson et al. 1989; Giorgi and Bates 1989; Mearns et al. 1995; Rummukainen et al. 2015; Takayabu et al. 2016). DS has several advantages over coarser global

\footnotetext{
${ }^{*}$ Correspondence: s-nishizawa@riken.jp
}

${ }^{1}$ RIKEN Advanced Institute for Computational Science, 7-1-26

Minatojima-minami-machi, Chuo-ku, Kobe, Hyogo 650-0047, Japan

Full list of author information is available at the end of the article simulations or reanalyses (e.g., Jones et al. 1995; Walsh and McGregor 1995; Giorgi and Shields 1999), e.g., better representation of surface conditions such as topography and land use, and utilization of more sophisticated physical process models such as a cloud microphysics model that avoids the need for cumulus parameterization.

For DS, one of the most important issues is which largescale state is used to drive the DS because the statistical characteristics of the downscaled state are significantly affected by the large-scale state. There are several DS methods in terms of derivation of the large-scale state. One such method is direct DS (DDS), where the largescale state obtained by a general circulation model or global climate model (GCM) is directly used. It has been pointed out that the climate bias in large-scale states has a significant impact on the reproducibility of the downscaled state (e.g., Kato et al. 2001; Wang et al. 2004). 
Another DS method is unbiased downscaling (UnbiasedDS), where the large-scale state obtained from a GCM experiment is used, as in DDS; however, the climate bias in the state from the GCM is removed. The climate bias in a GCM is often estimated as the difference between the climatology obtained by a GCM experiment under the current climate conditions and that obtained through reanalysis (e.g., Misra and Kanamitsu 2004; Holland et al. 2010; Done et al. 2015). Here, the model bias under future climate conditions is assumed to be identical to that under the present climate condition, although the bias may change under different climates (Bellprat et al. 2013). There is also an attempt to reduce the perturbation bias (e.g., Xu and Yang 2012; Jin et al. 2011). Another DS method is pseudo climate change downscaling (Pseudo-Clim-DS), which is also used to reduce the model climate bias, where the large-scale state is artificially constructed by adding a certain climate difference to the reanalysis state. An ideally constructed climate difference was used in Schär et al. (1996). The difference between the future projection and the present run in a GCM is also used as the climate difference (e.g., $\mathrm{Wu}$ and Lynch 2000; Kimura and Kitoh 2007; Sato et al. 2007; Cook and Vizy 2008; Kawase et al. 2009; Patricola and Cook 2010; Rasmussen et al. 2011), i.e., the climate bias in the model is treated in the same way as in Unbiased-DS. Wakazuki and Rasmussen (2015) constructed the climate difference statistically using multiple GCM simulations. Pseudo-Clim-DS attempts to reduce the perturbation bias using the perturbation value from the reanalysis. The validity of using the present perturbation in future DS projections was investigated by applying Pseudo-Clim-DS to a past climate in Kawase et al. (2008) and to an assumed true climate (ATC), i.e., the so-called perfect model experiment, in Yoshikane et al. (2012). Another advantage of the Pseudo-Clim-DS is that it allows us to investigate the influence of change in a target component on regional climate (e.g., Rowell and Jones 2006; Adachi et al. 2012; Kröner et al. 2016).

For future regional projections by DS, GCM results are utilized to obtain the large-scale state driving the DS. However, in GCMs, there are some inevitable limitations caused by model errors in terms of the obtained largescale states for DS. These limitations result in errors or uncertainties in the estimation of future regional change by DS, as well as uncertainties in future external conditions, such as in a scenario of greenhouse gas emission. A multi-model ensemble is a good strategy to evaluate the uncertainty, and much valuable knowledge has been obtained from ensemble experiments (e.g., van der Linden and Mitchell 2009; Means et al. 2013; Evans et al. 2014). However, uncertainties due to the limitations in individual models still have to be taken into account in order to achieve more reliable evaluations. Therefore, it is very important to consider the large-scale state obtained by GCM simulations in order to interpret the DS results. In this study, we consider the limitation from a possible error in the relationship between climatology and perturbations in GCM simulations through a consideration of the phase space of a large-scale state. On the basis of this consideration, we provide a comprehensive view of DS methods. We also propose a strategy for regional climate simulations with the goal of achieving better estimations and understanding of future changes in regional climate.

\section{Results}

In this section, we first explain the basic idea in this study, which is the decomposition of the large-scale state and the phase space spanned by the decomposed components. Next, we consider the uncertainties due to GCM errors and possible future states in the phase space. On the basis of this consideration, we propose a strategy for regional climate DS simulations in which various largescale states reconstructed from the decomposed components are used. Then, we present its advantages: a wider coverage of future possible states, better understanding of projected future change, and a comprehensive view of previous DS methods. Finally, we conduct a DS experiment to demonstrate how the strategy works to provide a better understanding by consideration of the individual influences of the decomposed components, which is one of the advantages.

\section{Decomposition of the large-scale state}

The large-scale state can be divided into multiple components. As one of the simplest decompositions, we consider climatology and the deviation from it (referred to as a perturbation) as two components.

Let the large-scale state be denoted as $\phi$, and its components of climatology and perturbation as $\langle\phi\rangle$ and $\phi^{\prime}$, respectively. There are several definitions of climatology, such as monthly long-term average. We discuss the definition in the "Discussion" section. Here, we consider states under climatological equilibrium. Considering different climates, the climatology is further decomposed into the reference climatology $\left\langle\phi_{0}\right\rangle$ and climate difference $\left\{\phi, \phi_{0}\right\}$ :

$$
\phi=\left\langle\phi_{0}\right\rangle+\left\{\phi, \phi_{0}\right\}+\phi^{\prime},
$$

where the climate difference is expressed as $\left\{\phi, \phi_{0}\right\}=$ $\langle\phi\rangle-\left\langle\phi_{0}\right\rangle$. For example, for future climate projections, the future atmospheric state $f$ can be decomposed into the present climatology $\langle p\rangle$, future climate difference $\{f, p\}$, and future perturbation $f^{\prime}$ so that $f=\langle p\rangle+\{f, p\}+f^{\prime}$.

Let us assume an extended phase space (referred to as just "phase space" in this paper) of a large-scale state. We define the state as having a temporal dimension; the total number of dimensions of the state vector in the phase space is obtained by multiplying the number of variables 
by the spatial degree of freedom and the temporal degree of freedom. A schematic diagram of the phase space is shown in Fig. 1. Note that, although the phase space has a large number of dimensions, the schematic diagram is drawn as two-dimensional (climatology and perturbation) by a dimensionality reduction, e.g., the principal component analysis, for ease of understanding. The horizontal axis represents the subspace $V_{\text {clim }}$, where $\langle\phi\rangle \in V_{\text {clim }}$, and the vertical axis represents the perpendicular complement space $V_{\text {clim }}^{\perp}$, where $\phi^{\prime} \in V_{\text {clim. }}^{\perp}$. In the phase space, a point corresponds to a state; state $\phi$ is located at point $\left(\langle\phi\rangle, \phi^{\prime}\right)$, e.g., the cross marks in Fig. 1.

\section{Uncertainty due to GCM errors}

The statistical characteristics of the climatology and perturbation are not independent of each other, since they are determined through their interactions with one another. Roughly speaking, they have a one-to-one correspondence, although there can be some ambiguities due to the possible existence of multi-equilibrium states. Here, we consider the statistical characteristics derived from the probability distribution function of possible states under the same climate condition, e.g., ensemble members in a GCM experiment; the characteristics of possible perturbations under a climate do not depend on individual realizations. The one-to-one correspondence means that states can exist only in a manifold in the entire phase space because they are constrained by their relationship, as indicated by the yellow lines in Fig. 1 .
None of the numerical models are perfect, and the representation of interaction among components depends on the GCMs. That is, their relationship in GCMs may be distorted from that in nature. For example, an aqua-planet intermodel comparison shows a variety of relationships: there exist significant differences in precipitation systems compared to the differences in mean flow (Blackburn et al. 2013). The former is part of the perturbation, and the latter is part of the climatology. Because of the distorted relationship in a GCM, the states in a numerical simulation may exist in a different manifold from that of nature, as shown in Fig. 2. One remarkable point here is that there is a significant probability of a real atmospheric state existing outside the region covered by numerical simulations in the phase space. DS driven only by the large-scale state in a distorted manifold can lead to an incorrect estimation of a regional climate.

Therefore, there is a need to explore a wider region than that covered by GCM simulations for the large-scale state driving the DS. The possible spuriousness in the relationship between the components in a GCM simulation is one of the causes of uncertainty in the estimation of the influence of climate change. If the GCM was perfect and we were confident about the perfection, the probability of the future state would be restricted only on the GCM's manifold, which is identical to that in nature. This results in good projections with smaller uncertainties. However, GCMs have errors; thus, the true state would be outside of the GCM's manifold because of the distorted constraint.

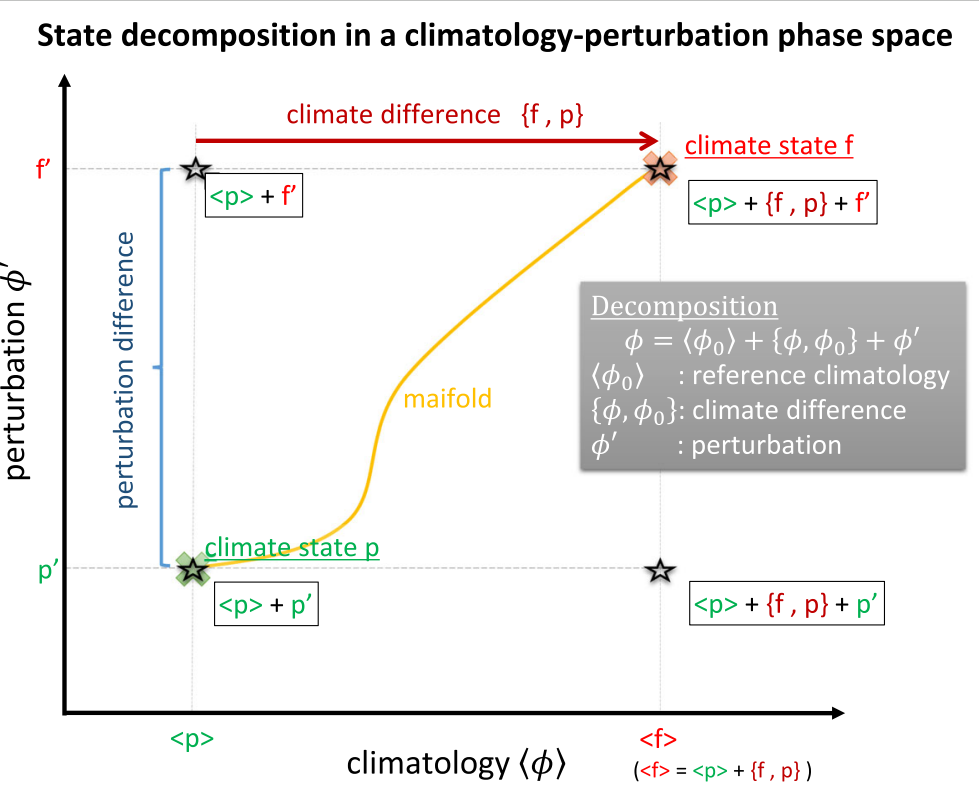

Fig. 1 Schematic diagram of the decomposition of the large-scale state in a climatology-perturbation phase space. Horizontal and vertical axes represent climatology and perturbation, respectively. For simplicity, climatology and perturbation are represented as scalar values. Cross marks show two states of a system. Stars represent the possible large-scale states driving the downscaling simulations. The yellow line shows the manifold corresponding to the relationship between climatology and perturbation 
Large scale state driving DS in the phase space for future projection

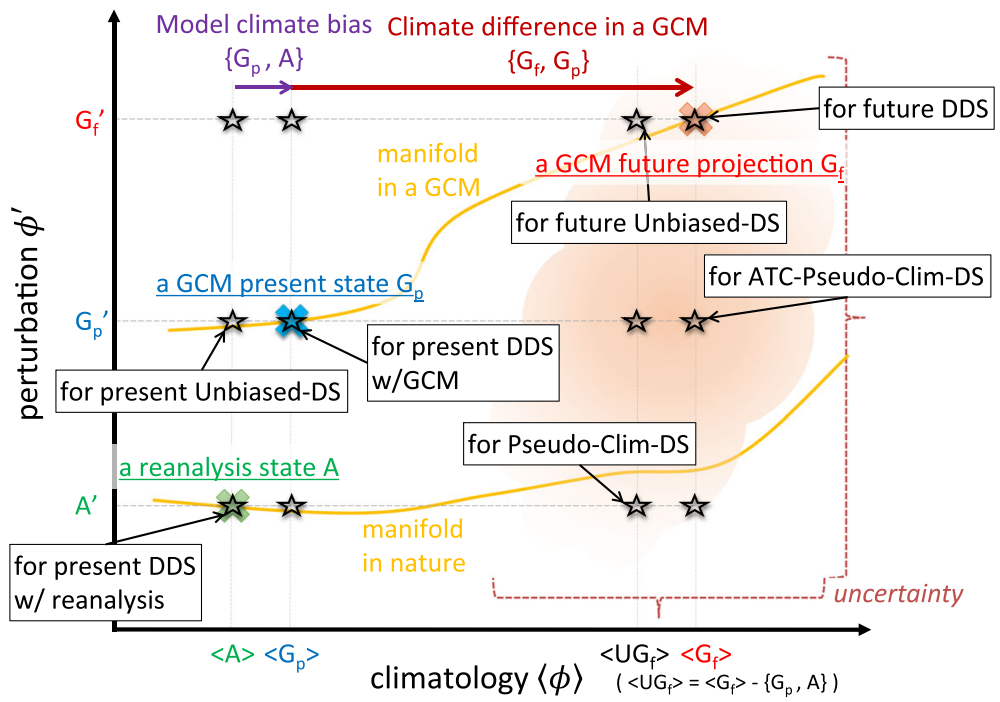

Fig. 2 Schematic diagram of the large-scale state driving the downscaling simulations. Cross marks show the states realized by reanalysis and GCM simulations. Stars represent the possible large-scale states driving the downscaling simulations. The orange shaded area represents the range of uncertainty in the estimation or existence probability distribution of the future state. The yellow line shows the manifold corresponding to the relationship between climatology and perturbation

That is, the possible GCM errors spread the probability distribution. The region covered by simulations with a GCM in the phase space is limited to the spurious manifold. In fact, the trends in the CMIP5 historical runs (Taylor et al. 2012) are overconfident, and the observed trend is outside the range of the multi-model ensembles (van Oldenborgh et al. 2013). The bias-correcting methods produce states away from the GCM manifold, but the methods only shift the region in the phase space to another place and do not enlarge the region to be explored.

\section{Reconstruction of large-scale states}

In order to obtain large-scale states that are outside of the GCM's manifold, we reconstruct the large-scale states. The climatology and perturbation from different states are not constrained in the distorted relationship of GCMs. Therefore, reconstruction by combining them produces large-scale states that are outside of the manifold. Let us consider the case of present and future states. Two components, the climatology and perturbations of the two states, are taken into account (Fig. 1). Considering these as independent components, four large-scale states can be obtained through their combinations, as illustrated by the stars in the diagram. It can be seen that two of these states are away from the spurious constraint. This independent treatment of the components achieves evaluation of states in a wider region of the phase space than the manifold of a GCM. The technical procedure to reconstruct the largescale state is based on the Pseudo-Clim-DS in terms of combining components from different states. However, in this conceptualization, the large-scale states are obtained in multiple ways. The previous DS methods, such as DDS, Unbiased-DS, and Pseudo-Clim-DS, are comprehensively involved.

For practical purposes, we often have three large-scale states: a reanalysis of the present climate state $A$, a GCM of the present climate state $G_{p}$, and a GCM of the future climate state $G_{f}$ (Fig. 2). Further, there are four climatologies: $\langle A\rangle,\left\langle G_{p}\right\rangle,\left\langle G_{f}\right\rangle$, and $\left\langle U G_{f}\right\rangle$. The last one is the unbiased future GCM climatology in which the climate bias of the model estimated under the present climate is removed:

$$
\left\langle U G_{f}\right\rangle=\left\langle G_{f}\right\rangle-\left\{G_{p}, A\right\}=\langle A\rangle+\left\{G_{f}, G_{p}\right\} .
$$

For perturbations, there can be three states: $A^{\prime}, G_{p}^{\prime}$, and $G_{f}^{\prime}$. Thus, in total, there exist 12 possible states for DS, which are indicated by stars in the diagram. More possible states can be also obtained through combinations with other components, such as the ones obtained by ensemble GCM simulations and/or artificially generated or modified states. These states are widely spread in the phase space and are not limited by the GCM constraint. Here, we should take into account the consistency between the combined components in the combinations. Statistical checking of the consistency in spatial and temporal aspects is an important issue. At least, we have to check whether certain phenomena in the constructed state are consistent with our knowledge. For instance, the location of the mid-latitude westerly jet (i.e., a horizontal temperature gradient) and that of baroclinic eddies should 
be consistent, and tropical cyclones (TCs) should not be produced on the dry side of the Baiu front.

Our concept has another advantage in addition to wider coverage of the large-scale state in the phase space. The influence of changes in the individual components on the downscaled regional states can be examined. As a typical example, let us assume the following case: each difference in the climatology and perturbation between the two states in the spurious manifold has opposite influences on the regional climate from each other, but not in nature's manifold. In this case, the DS would lead to an underestimation of the total influence. The above discussion indicates the importance of evaluating individual influences in order to consider the total influence. Through a comparison of multiple DS simulations driven by the large-scale states that differ from one another by only one component, the influence of the individual components can be estimated. The regional state $\psi$ obtained by DS depends on the large-scale state $\phi: \psi=\mathcal{D}\left[\phi^{\prime},\langle\phi\rangle\right]$, where $\mathcal{D}$ is an operator representing the DS. The operator is interpreted as

$$
\psi=\mathcal{D}\left[\phi^{\prime},\langle\phi\rangle\right]=\langle\phi\rangle+\mathcal{P}\left[\phi^{\prime} ;\langle\phi\rangle\right]+\mathcal{G}[\phi],
$$

where $\mathcal{P}$ is an operator representing modification of the large-scale perturbation $\phi^{\prime}$ under climatology $\langle\phi\rangle$ by DS and $\mathcal{G}$ is one representing the variability generated or excited spontaneously under the given large-scale environment in the DS simulation. $\mathcal{G}$ consists of sub-GCMgrid-scale variability, which does not get resolved in GCM simulations, but gets resolved in DS simulations. The differences in the downscaled results with the different states, $\mathcal{D}\left[f^{\prime},\langle f\rangle\right]-\mathcal{D}\left[p^{\prime},\langle p\rangle\right]$, can be divided into
$\mathcal{D}\left[p^{\prime},\langle p\rangle+\{f, p\}\right]-\mathcal{D}\left[p^{\prime},\langle p\rangle\right]$ and $\mathcal{D}\left[f^{\prime},\langle p\rangle+\{f, p\}\right]-$ $\mathcal{D}\left[p^{\prime},\langle p\rangle+\{f, p\}\right]$. The first represents the difference due to the change in climatology, and this is the quantity estimated with Pseudo-Clim-DS. This can be mainly interpreted as the difference in the intensification and/or moving speed of a given perturbation under different climatologies in the DS simulations. The second represents the difference due to the difference in a given perturbation under the same climatology, e.g., the influence of the number of cyclones passing through the calculation domain.

The difference in the existing DS methods, clarified through our concept of constructing the large-scale state from independent components as their decomposition, is summarized in Table 1. In addition, the location of the states in the phase space is illustrated in Fig. 2. The concept can be described as a generalization of existing DS methods. Comparisons of the results obtained by different existing DS methods can be used to estimate the individual influence of the components. The influence of the difference in climatology can be estimated by comparing the results obtained by DDS driven by reanalysis data and Pseudo-Clim-DS. A comparison can also be made between DDS driven by present climate GCM data and ATC-Pseudo-Clim-DS. The influence of perturbation can be examined by performing a comparison between the following DSs cases: (i) DDS with reanalysis data and present climate Unbiased-DS, (ii) future climate DDS and ATC-Pseudo-Clim-DS, and (iii) future climate UnbiasedDS and Pseudo-Clim-DS. A comprehensive evaluation of the results obtained by different DS methods in previous studies helps us understand the contribution of various

Table 1 Possible large-scale states driving downscaling with reanalysis and GCM simulations in present and future climates

\begin{tabular}{|c|c|c|c|}
\hline Perturbation & $A^{\prime}$ & $G_{p}^{\prime}$ & $G_{f}^{\prime}$ \\
\hline \multicolumn{4}{|l|}{ Climatology } \\
\hline$\langle A\rangle$ & $A$ & $G_{p}-\left\{G_{p}, A\right\}$ & \\
\hline \multirow[t]{2}{*}{$(=\langle A\rangle+\{A, A\})$} & $\langle A\rangle+\{A, A\}+A^{\prime}$ & $\langle A\rangle+\{A, A\}+G_{p}^{\prime}$ & $\langle A\rangle+\{A, A\}+G_{f}^{\prime}$ \\
\hline & Present DDS (reanalysis) & Present Unbiased-DS & \\
\hline$\left\langle G_{p}\right\rangle$ & & $G_{p}$ & \\
\hline \multirow[t]{2}{*}{$\left(=\langle A\rangle+\left\{G_{p}, A\right\}\right)$} & $\langle A\rangle+\left\{G_{p}, A\right\}+A^{\prime}$ & $\langle A\rangle+\left\{G_{p}, A\right\}+G_{p}^{\prime}$ & $\langle A\rangle+\left\{G_{p}, A\right\}+G_{f}^{\prime}$ \\
\hline & & Present DDS (GCM) & \\
\hline$\left\langle U G_{f}\right\rangle$ & $A+\left\{G_{f}, G_{p}\right\}$ & & $G_{f}-\left\{G_{p}, A\right\}$ \\
\hline \multirow[t]{2}{*}{$\left(=\langle A\rangle+\left\{G_{f}, G_{p}\right\}\right)$} & $\langle A\rangle+\left\{G_{f}, G_{p}\right\}+A^{\prime}$ & $\langle A\rangle+\left\{G_{f}, G_{p}\right\}+G_{p}^{\prime}$ & $\langle A\rangle+\left\{G_{f}, G_{p}\right\}+G_{f}^{\prime}$ \\
\hline & Pseudo-Clim-DS & & Future Unbiased-DS \\
\hline$\left\langle G_{f}\right\rangle$ & & $G_{p}+\left\{G_{f}, G_{p}\right\}$ & $G_{f}$ \\
\hline \multirow[t]{2}{*}{$\left(=\langle A\rangle+\left\{G_{f}, A\right\}\right)$} & $\langle A\rangle+\left\{G_{f}, A\right\}+A^{\prime}$ & $\langle A\rangle+\left\{G_{f}, A\right\}+G_{p}^{\prime}$ & $\langle A\rangle+\left\{G_{f}, A\right\}+G_{f}^{\prime}$ \\
\hline & & ATC-Pseudo-Clim-DS & Future DDS \\
\hline
\end{tabular}

The top, middle, and bottom rows in each cell represent the original form, decomposition form, and corresponding downscaling method, respectively. The climatology and perturbation of a state are represented by \langle\rangle and prime, respectively, and the climate difference by \{ \} 
components to the total regional climate change in the future.

\section{Demonstration of individual influences}

In order to demonstrate an advantage of our concept, that is, the importance of estimating the individual influences of changes in climatology and perturbation in the large-scale state on regional precipitation, we conduct a DS experiment based on the decomposition concept as an example. We simultaneously consider the influences of the two components on regional precipitation through a comparison of three DS simulations. The experimental design is essentially the same as that of Yoshikane et al. (2012). However, our interest here is to focus on the decomposition of the total influence into those of the climatology and perturbation, whereas the purpose of Yoshikane's study was to verify the validity of the Pseudo-Clim-DS method for future DS projection.

The three DSs, i.e., the present climate DDS (PresentDDS) run, the future climate DDS (Future-DDS) run, and the ATC-Pseudo-Clim-DS run, are performed. The largescale state driving the DS are denoted by $G_{p}, G_{f}$, and $G_{p}+\left\{G_{f}, G_{p}\right\}$ for the Present-DDS, Future-DDS, and ATCPseudo-Clim-DS runs, respectively, where the states for the present and future climates in the GCM experiment are denoted by $G_{p}$ and $G_{f}$, respectively. These can be written as follows in the decomposed form:

$$
\begin{aligned}
G_{p} & =\left\langle G_{p}\right\rangle+\left\{G_{p}, G_{p}\right\}+G_{p}^{\prime}, \\
G_{f} & =\left\langle G_{p}\right\rangle+\left\{G_{f}, G_{p}\right\}+G_{f}^{\prime}, \\
G_{p}+\left\{G_{f}, G_{p}\right\} & =\left\langle G_{p}\right\rangle+\left\{G_{f}, G_{p}\right\}+G_{p}^{\prime} .
\end{aligned}
$$

The influence of climatology is estimated from the difference between the ATC-Pseudo-Clim-DS and Present-DDS runs, $\mathcal{D}\left[G_{p}^{\prime},\left\langle G_{p}\right\rangle+\left\{G_{f}, G_{p}\right\}\right]-\mathcal{D}\left[G_{p}^{\prime},\left\langle G_{p}\right\rangle\right]$, and that of the perturbation from the difference between the Future-DDS and ATC-Pseudo-Clim-DS runs, $\mathcal{D}\left[G_{f}^{\prime},\left\langle G_{p}\right\rangle+\left\{G_{f}, G_{p}\right\}\right]-\mathcal{D}\left[G_{p}^{\prime},\left\langle G_{p}\right\rangle+\left\{G_{f}, G_{p}\right\}\right]$.

In this demonstration, experiments involving 25-yearlong time slices for the summer season (from June to September; referred to as JJAS) were performed under the present (1979 to 2003) and future (2075 to 2099) climate conditions in East Asia. Detailed descriptions of the experiment and the model are in the "Methods/Experimental" section. In the demonstration, the daily mean is used as the climatology of the largescale state. We first obtained the monthly climatology by 25 -year averaging. Next, we assumed that the monthly mean is the value on the 15th of each month and constructed the daily climatology by linear interpolation of the monthly climatology. Here, we assume that climate change over 25 years is small enough to consider that the state is in climatological equilibrium. We ignore the bias in order to focus on demonstrating how individual influences can be considered with the concept, although a formal assessment of future regional climate will need to consider the model climate bias. The analysis is conducted with the simulation result in domain 2 described in the "Methods/Experimental" section, except for the lateral nudging region.

Figure 3 shows the summer climatology of the daily precipitation in the Present-DDS, ATC-Pseudo-Clim-DS, and Future-DDS runs. A large amount of precipitation is found in the high mountain areas near the Pacific Ocean. The precipitation in the Future-DDS is significantly weaker than that in the Present-DDS for the highprecipitation areas and the Pacific (Fig. 3f). The difference in precipitation resulting from climatology (Fig. 3d) is much smaller than the total difference. On the other hand, the difference resulting from perturbation is statistically significant (Fig. 3e). In another analysis by month, we confirmed significant differences due to perturbation in all months from June to September. This shows that the differences due to perturbation result in significant differences in the DS simulations. Domain-averaged precipitation shows the same tendency (Fig. 4). The influences of climatology and perturbation have opposite signs. Since the individual influences have uncertainties, the magnitude of each estimated change must be considered with some range of the uncertainty. In our result, the positive change due to the climate difference is smaller than the negative change due to the perturbation difference in terms of absolute magnitude. Because of the uncertainty, there is a possibility that the magnitude relationship may reverse; that is, the positive change due to the climate difference might be larger than the negative change due to the perturbation difference. Therefore, although the total difference in precipitation has a negative value in this experiment, it is possible for the total difference to be positive. This suggests that interpretations made by considering only the total change or the change in one component face the risk of misunderstanding the future regional climate. A simultaneous estimation considering the total and individual components is thus necessary. This result demonstrates the usefulness of the decomposition concept we propose in this study.

In order to show the usefulness of decomposition in understanding the future precipitation change, we conduct further decomposition of TC and non-TC precipitations. This can help readers in understanding the availability of our proposed concept by showing the series of our research process of the regional climate change. Although this decomposition is not directly specified by the proposed concept of DS, it strengthens our premise that decomposition is a useful way to understand future changes through understanding of individual influences of decomposed components on the future change. TCs have a large impact on precipitation in 


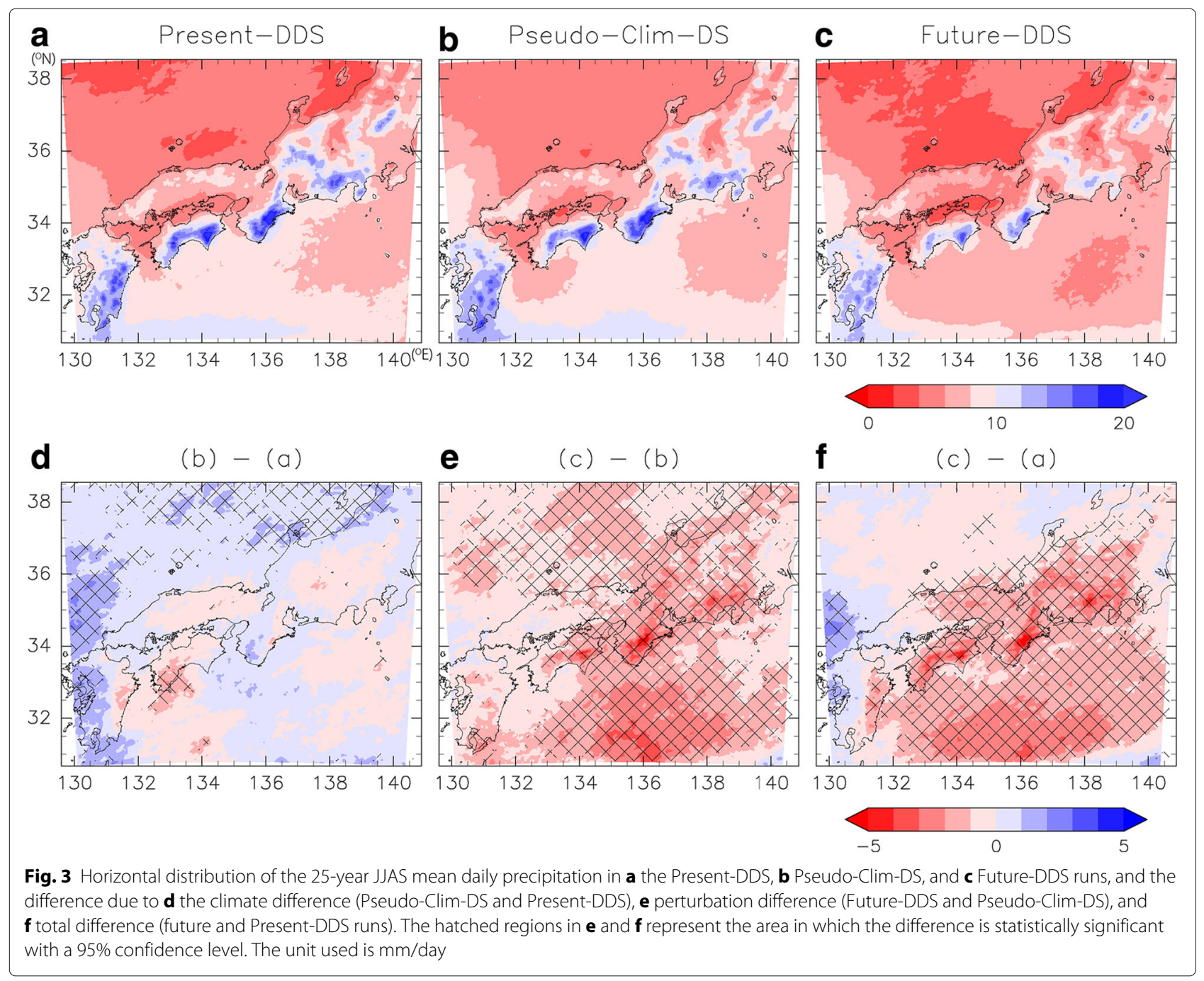

this region. In the GCM future projection run used to drive the DS, the number of TCs is smaller than that in the present run (Murakami et al. 2012). The fewer TCs in future climate is also indicated by previous studies using other GCMs (e.g., Knutson et al. 2015; Roberts et al. 2015). The smaller number of TCs in the future is consistent with the smaller amount of precipitation. We examine the precipitation associated with the TCs. Cyclone track detection is described in the "Methods/Experimental" section. We define TC precipitation as the precipitation within a distance of $1000 \mathrm{~km}$ from the center of a TC. The contribution of the TC precipitation to the total precipitation change is larger than that of the non-TC precipitation, as shown in Fig. 4 (top). The precipitation change associated with TCs is primarily attributed to the perturbation difference. This is consistent with the fact that the number of TCs projected in the future perturbation is much smaller than that in the present. Increasing TC precipitation due to the climate difference can be observed (Fig. 4, middle). This implies that TCs are intensified in the future climatology compared to the present. This result is consistent with previous studies (e.g., Ying et al. 2012; Christensen et al. 2013). It is observed that the decreasing trend in the nonTC precipitation is mainly attributed to the perturbation difference. The change is dominant in June, and it is not as large in other months. The smaller non-TC precipitation in June is consistent with the slower northward movement of the Baiu front in the GCM future projection than in the present (Kusunoki et al. 2011; Kanada et al. 2012). On the other hand, the influence of climatology on the non-TC precipitation is quite small.

\section{Discussion}

Different areas, seasons, and quantities, i.e., different variables and statistics, can show the different contributions of individual components to regional climate change. For instance, the $2 \mathrm{~m}$ temperature in the experiment shows the 


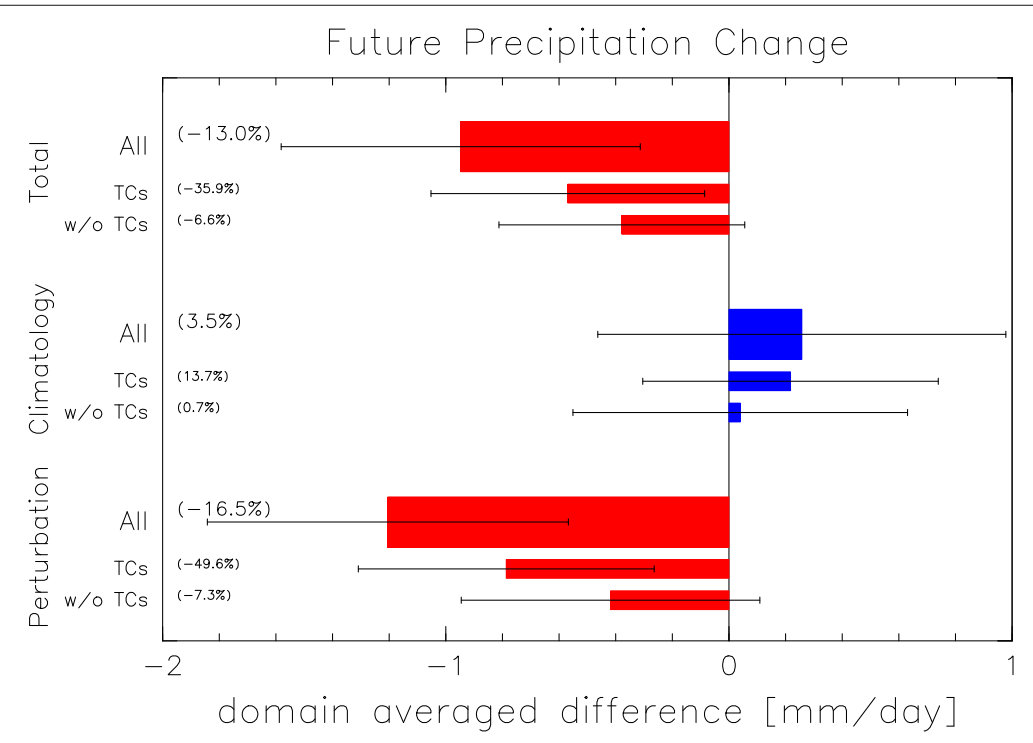

Fig. 4 Contribution of each component to domain-averaged 25-year JJAS mean daily precipitation difference. The total difference, the difference due to climate difference, and that due to perturbation are shown at the top, middle, and bottom, respectively, on the vertical axis. These differences are further divided into ones associated with tropical cyclones and other factors. The error bars represent the $95 \%$ confidence interval of Welch's statistics. The percentages represent the ratios of the difference to the total precipitation in the Present-DDS run

dominant contribution of the climate difference relative to the difference in the perturbation as shown in Fig. 5, and the perturbation difference is dominant in the precipitation change. This suggests that applying this concept to various cases and fields is important in order to interpret the regional future projections.

In the analysis discussed in the demonstration, nonlinear interaction between climatology and perturbation in the DS simulation, i.e., nonlinearity in operator $\mathcal{D}$, is not considered. Actually, operator $\mathcal{D}$ has some degree of nonlinearity, and the estimation of individual influences would be contaminated because of the nonlinearity. The comparison between $\mathcal{D}\left[p^{\prime},\langle p\rangle+\{f, p\}\right]-\mathcal{D}\left[p^{\prime},\langle p\rangle\right]$ and $\mathcal{D}\left[f^{\prime},\langle p\rangle+\{f, p\}\right]-\mathcal{D}\left[f^{\prime},\langle p\rangle\right]$ or $\mathcal{D}\left[f^{\prime},\langle p\rangle\right]-\mathcal{D}\left[p^{\prime},\langle p\rangle\right]$ and $\mathcal{D}\left[f^{\prime},\langle p\rangle+\{f, p\}\right]-\mathcal{D}\left[p^{\prime},\langle p\rangle+\{f, p\}\right]$ gives us a hint of the nonlinearity. The nonlinearity can also be investigated by comparing multiple DS simulations based on the strategy. Adachi et al. (2017) proposed a procedure to extract the interaction effect and showed that the interaction can have a greater influence on heavier precipitation, whereas Kröner et al. (2016) showed that the nonlinearity could be negligible in their case. Careful considerations for the nonlinearity are necessary in each case.

In the DS experiment in this study, climatology is defined as the daily interpolated climatological monthly mean. Interannual and inter-seasonal variability, such as ENSO, is included in the perturbation. There are variations in the definition of climatology. For instance, although seasonal and daily changes can be included in climatology, they can also be treated as perturbations.
These components should be defined based on the purpose of the study. In addition, the climatologyperturbation decomposition is just one of several possible decompositions. In order to understand the influences of other components, different decompositions would be appropriate. For instance, Adachi et al. (2012) investigated the impact of urbanization on regional climate with a Pseudo-Clim-DS experiment with the present and future urbanization conditions. Kröner et al. (2016) decomposed the future change into changes due to three effects (the large-scale thermodynamic effect, lapse-rate effect, and large-scale circulation) and other remaining effects. Comparisons between the individual impacts obtained through such experiments would give us a better understanding of future change. Here, it should be noted that dynamical or other balances should be considered in the independent treatment of the decomposed components; otherwise, the variability in DS simulations could be violated because of non-physical factors. In addition, we consider that other quantities (not just the mean) are important in climate change, such as quantities related to extremes, although we discuss only the mean as an example.

We focus on the GCM's errors in this study. There also exist model errors in regional climate models (RCMs), which represent the operator $\mathcal{D}$ in Eq. 3, that can be a cause of uncertainty as well (e.g., Déqué et al. 2012). The $\mathrm{RCM}$ error can be interpreted as two errors. One error is that in the operator $\mathcal{P}$ and is caused by the spurious modification of the large-scale perturbation. The other is that in the operator $\mathcal{G}$ and is caused by the spurious excitation 


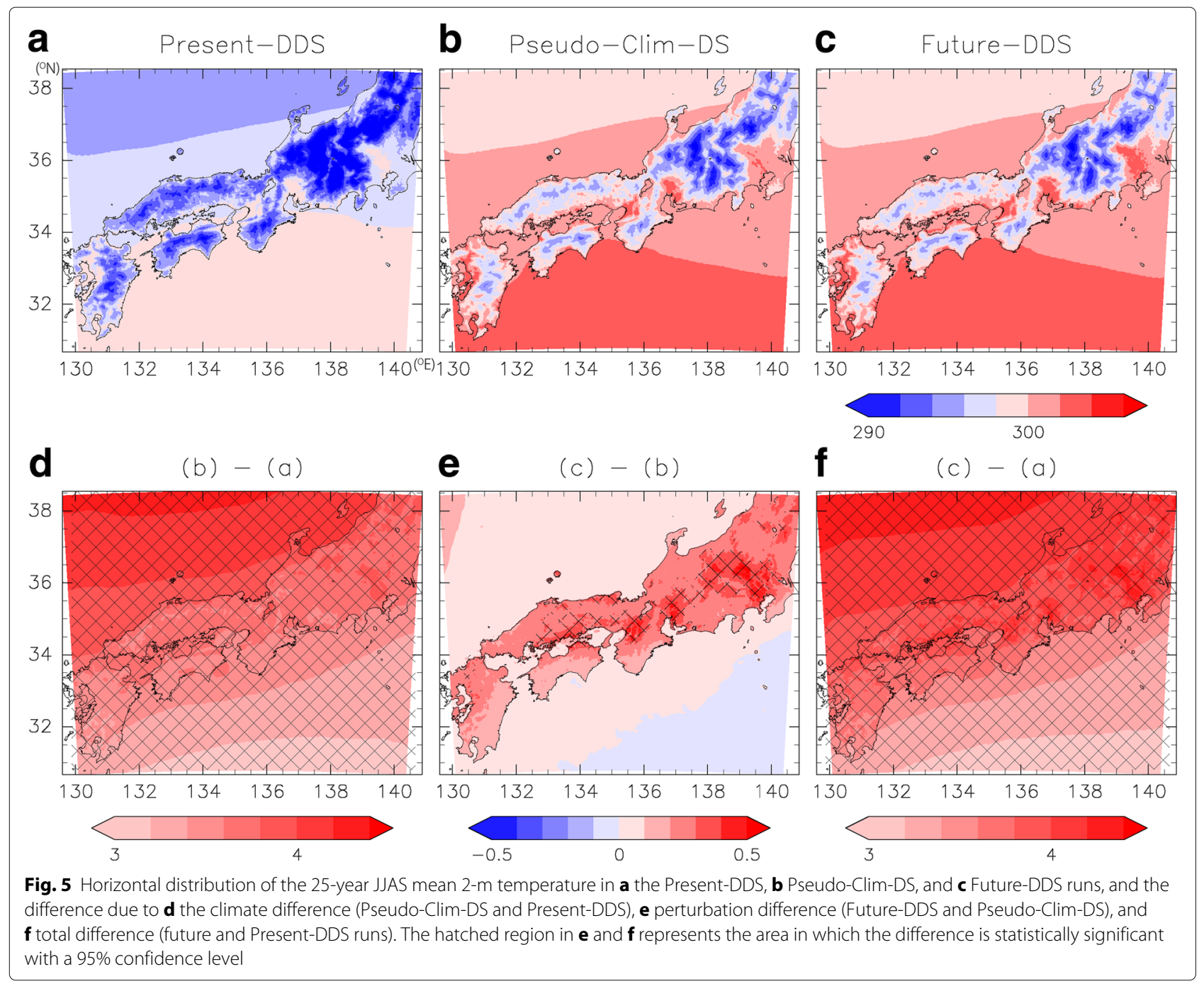

of small-scale variability. In addition to considering the uncertainty due to the GCM's errors by including a wider coverage of possible large-scale states, it is important to consider the uncertainty due to the RCM's errors, which can be evaluated by multi-RCM experiments.

\section{Conclusions}

We conceptualized dynamical downscaling and presented a perspective on downscaling for regional climate studies. In this study, we provided a comprehensive view of the previous downscaling methods based on reconstruction of the large-scale state driving DS from independent decomposed components, such as climatology and perturbation.

The concept of the reconstruction of the decomposed components provides not only a comprehensive view of DS but also a strategy for coordinated experimental design for future regional climate studies. We can obtain the large-scale states from a wider region in the phase space, whereas only the states in the spurious manifold in the phase space distorted by interactions between the components due to a GCM error are obtained by the previous individual DS methods. Furthermore, we can estimate the individual influences of the change in the components of the large-scale state on the downscaled regional climate. The importance of the simultaneous consideration of individual influences is demonstrated by a DS experiment. We estimated the influence of both the climatology and perturbation change on the future precipitation simultaneously and found that the influence of the perturbation can be significant, as well as that of the climatology.

Consideration of uncertainty due to the model error and understanding of the influence of individual components are important for more reliable assessments of future regional climates. The uncertainty of the projected future regional climate is often estimated from an ensemble spread in ensemble experiments with various GCM 
results. The region in the phase space covered by multimodel and/or multi-physics ensemble GCM simulations is larger than that of a single-model and single-physics case. However, the manifolds of the different GCMs can be isolated from one another, and the covered region was discretely distributed. In order to understand the characteristics of the output of a nonlinear operator, inputs that are not only widely but also evenly distributed are generally preferable to those that are locally concentrated in some places because the operator has local linearity. In this sense, in order to assess the uncertainty more reliably, DS simulations driven not only by the large-scale states obtained by ensemble GCM simulations but also by reconstructed states from components of the ensembles should be performed. In addition to the total uncertainty, an estimation of the individual uncertainty associated with each component is as necessary as the absolute magnitude of the influence due to individual components. The contribution of each component differs in its uncertainty and absolute magnitude.

\section{Methods/Experimental}

\section{Experimental settings}

In the demonstration, the total 25-year summer time data was obtained by performing five-day integrations overlapping a one-day spin-up term. Two nested domains are used for DS: domain 1, which is $2520 \times 2520 \mathrm{~km}^{2}$ with a $7.5 \mathrm{~km}$ grid spacing, and domain 2 nested in domain 1 , which is $1080 \times 960 \mathrm{~km}^{2}$ with a $2.5 \mathrm{~km}$ grid spacing; both domains have the same domain center of $135.2^{\circ} \mathrm{E}$ and $34.7^{\circ} \mathrm{N}$ (Fig. 6) The numbers of vertical layers are 36 and 60 for domains 1 and 2, respectively.
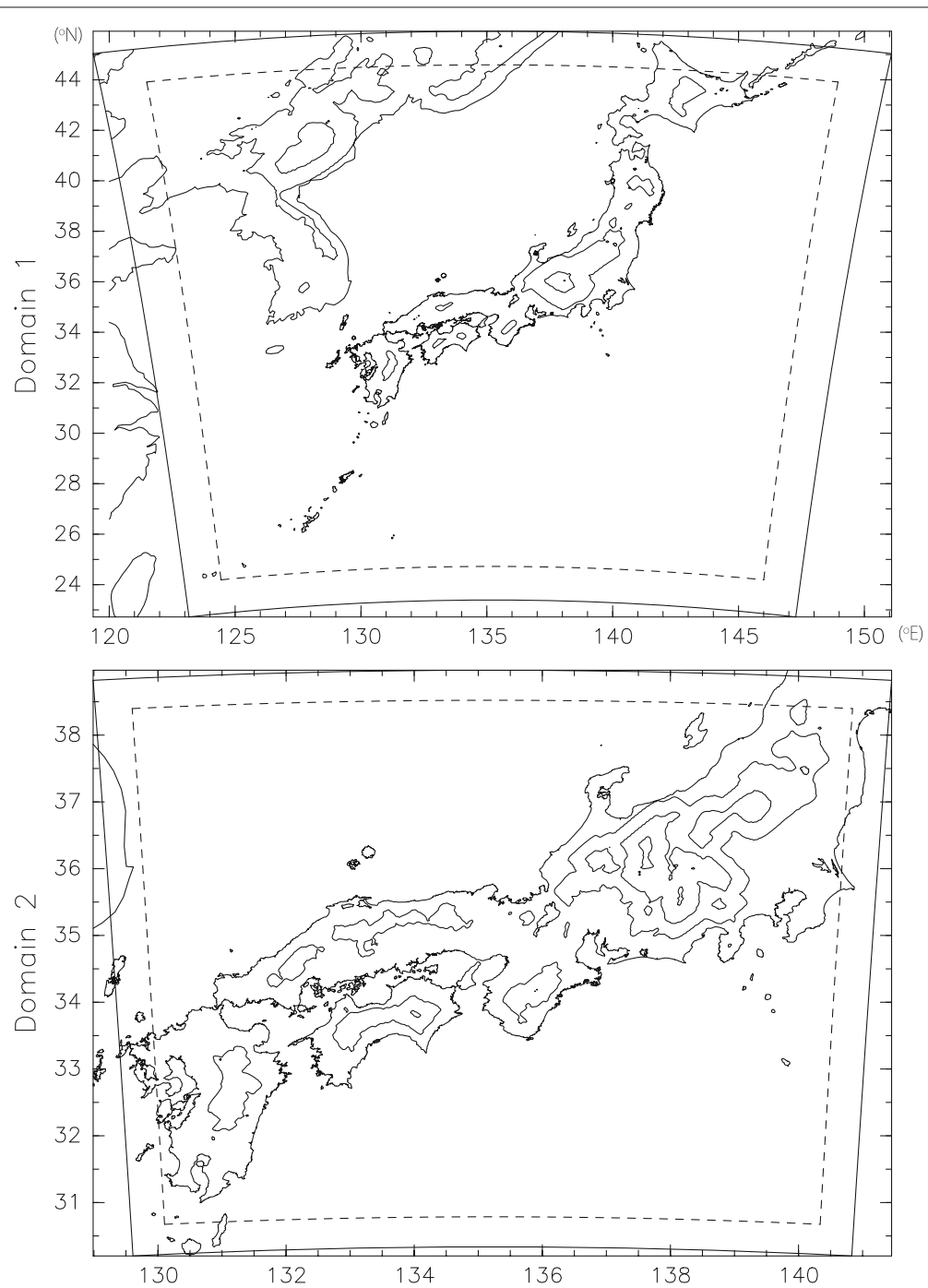

Fig. 6 Domain (top) 1 and (bottom) 2 for the numerical simulation in the demonstration. Contours represent surface elevation in the simulation and their interval is $500 \mathrm{~m}$. The broken line shows the lateral nudging region (outmost 20 grids) 


\section{Regional model}

The regional model used for the DS experiment is the Scalable Computing for Advanced Library and Environment Regional model (SCALE-RM) (Nishizawa et al. 2015; Sato et al. 2015; available at http://scale.aics.riken.jp). A six-category one-moment bulk scheme (Tomita 2008), a $k$-distribution scheme (Sekiguchi and Nakajima 2008), the MYNN level 2.5 scheme (Nakanishi and Niino 2009), a similarity scheme (Beljaars and Holtslang 1991; Wilson 2001), a diffusion and bucket scheme, and a single-layer urban canopy model (Kusaka et al. 2001) are used to represent cloud microphysical processes, radiation transfer, planetary boundary layer turbulence, surface flux, land processes, and urban processes, respectively.

\section{Large-scale state}

The large-scale state obtained by a $20-\mathrm{km}$ resolution Atmospheric Model Intercomparison Project (AMIP)type experiment with the Meteorological Research Institute atmospheric GCM (MRI-AGCM) (Mizuta et al. 2012) is used to drive the DS. In the AGCM experiment, the sea surface temperature (SST) of HadISST1 (Rayner et al. 2003) is prescribed in the present climate run (Mizuta et al. 2012), and the multi-model ensemble averaged SST of the CMIP5 (Taylor et al. 2012) coupled-GCM runs with the Representative Concentration Pathways (RCP) 8.5 scenario is prescribed in the future climate run (Mizuta et al. 2014).

\section{Cyclone track detection}

The cyclone track is detected using the method proposed by Adachi and Kimura (2007), and a TC is defined as a cyclone that comes from south of $25^{\circ} \mathrm{N}$. The corresponding cyclones in the GCM simulation are used for the TC determination, since the domain for the DS is not wide enough for the determination. The numbers of TCs entering the domain of analysis are 150, 158, and 90, in the Present-DDS, ATC-Pseudo-Clim-DS, and Future-DDS runs, respectively. Here, the numbers are different for the Present-DDS and ATC-Pseudo-Clim-DS runs because of slight differences in TC tracks in the outer domain.

\section{Abbreviations \\ AGCM: Atmospheric general circulation model; AMIP: Atmospheric model intercomparison project; ATC: Assumed true climate; DS: Downscaling; DDS: Direct downscaling; GCM: General circulation model or Global climate model; JJAS: June to September; MRI: Meteorological Research Institute; \\ Pseudo-Clim-DS: Pseudo climate change downscaling; RCM: Regional climate model; SST: Sea surface temperature; TC: Tropical cyclones}

\section{Acknowledgements}

The MRI-AGCM3.2S data were provided by the Program for Risk Information on Climate Change (SOUSEI) of the Ministry of Education, Culture, Sports, Science, and Technology (MEXT) of Japan conducted by the Meteorological Research Institute (MRI). This research used computational resources of the K computer provided by the RIKEN Advanced Institute for Computational Science through the HPCI System Research project (Project ID:hp160119). The analysis and visualization in this study was conducted using the products of the GFD-Dennou Club (http://www.gfd-dennou.org/).

\section{Funding}

This work was supported by the FOCUS Establishing Supercomputing Center of Excellence and JST CREST Grant Number JPMJCR1312, Japan.

\section{Availability of data and materials}

The regional model for the downscaling calculation in this study was constructed using SCALE, which is freely available at https://scale.aics.riken.jp/ download/index.html under the 2-Clause BSD license. The model code used is based on SCALE-RM version 4.2.5 including some improvements such as the usability of the microphysics scheme. The model code and the set of configuration parameters are available from the corresponding author upon reasonable request. The pre-process code that generates the boundary conditions for SCALE-RM from the MRI-AGCM3.2 data and the post analysis code used in this study are also available from the corresponding author. The downscaling data by SCALE-RM is deposited in local storage at RIKEN/AICS. It is available from the corresponding author upon reasonable request. The MRI-AGCM data was provided to the authors by MRI under a given condition that the authors can use the MRI-AGCM data only for the current and related studies. The data is not publicly available. However, upon reasonable request with regards to this study, the data are provided from MRI with the permission of MRI. In this case, contact person is the corresponding author.

\section{Authors' contributions}

SN, SAA, YK, and HT designed the research. SN, SAA, TY, and KA carried out the downscaling simulation. SN, SAA, TY, KA, and RY analyzed the data. SN, SAA, YK, and HT wrote the paper. YK, HY, and HT supervised the research. All authors contributed to discussions of the results and read and approved the manuscript.

\section{Ethics approval and consent to participate}

Not applicable.

\section{Consent for publication \\ Not applicable.}

\section{Competing interests}

The authors declare that they have no competing interests.

\section{Publisher's Note}

Springer Nature remains neutral with regard to jurisdictional claims in published maps and institutional affiliations.

\section{Author details}

${ }^{1}$ RIKEN Advanced Institute for Computational Science, 7-1-26 Minatojima-minami-machi, Chuo-ku, Kobe, Hyogo 650-0047, Japan. ${ }^{2}$ Research Center for Urban Safety and Security, Kobe University, 1-1 Rokkodai-cho, Nada-ku, Kobe, Hyogo 657-8501, Japan.

Received: 8 May 2017 Accepted: 13 December 2017

Published online: 08 January 2018

\section{References}

Adachi SA, Kimura F (2007) A 36-year climatology of surface cyclogenesis in East Asia using high-resolution reanalysis data. SOLA 3:113-116. doi:10.2151/sola.2007-029

Adachi SA, Kimura F, Kusaka H, Inoue T, Ueda H (2012) Comparison of the impact of global climate changes and urbanization on summertime future climate in the Tokyo metropolitan area. J Appl Meteorol Climatol:1441-1454. doi:10.1175/JAMC-D-11-0137.1

Adachi SA, Nishizawa S, Yoshida R, Yamaura T, Ando K, Yashiro H, Kajikawa Y, Tomita H (2017) Contributions of changes in climatology and perturbation and the resulting nonlinearity to regional climate change. Nat Commun 8(2224). doi:10.1038/s41467-017-02360-z

Beljaars ACM, Holtslag AAM (1991) Flux parameterization over land surfaces for atmospheric models. J Appl Meteorol 30:327-341. http://doi.org/10.1175/ 1520-0450(1991)030\%3C0327:FPOLSF\%3E2.0.CO;2

Bellprat O, Kotlarski S, Lüthi D, Schär C (2013) Physical constraints for temperature biases in climate models. Geophys Res Lett 40:4042-4047. doi:10.1002/grl.50737 
Blackburn M, Williamson DL, Nakajima K, Ohfuchi W, Takahashi YO, Hayashi Y-Y, Nakamura H, Ishiwatari M, McGregor JL, Borth H, Wirth V, Frank H, Bechtold P, Wedi NP, Tomita H, Satoh M, Zhao M, Held IM, Suarez MJ, Lee M-I, Watanabe M, Kimoto M, Liu Y, Wang Z, Molod A, Rajendran K, Kitoh A, Stratton R (2013) The aqua-planet experiment (APE): Control SST simulation. J Meteorol Soc Japan Ser II 91A:17-56. doi:10.2151/jmsj. 2013-A02

Christensen JH, Kumar KK, Aldrian E, An S-I, Cavalcanti IFA, de Castro M, Dong W, Goswami P, Hall A, Kanyanga JK, Kitoh A, Kossin J, Lau N-C, Renwick J, Stephenson DB, Xie S-P, Zhou T (2013) Climate phenomena and their relevance for future regional climate change. In: Climate Change 2013: The Physical Science Basis. Contribution of Working Group I to the Fifth Assessment Report of the Intergovernmental Panel on Climate Change. Cambridge University Press, Cambridge. Chap. 14

Cook KH, Vizy EK (2008) Effects of twenty-first-century climate change on the Amazon rain forest. J Climate 21:542-560. doi:10.1175/2007JCLI1838.1

Déqué M, Somot S, Sanchez-Gomez E, Goodess CM, Jacob D, Lenderink G, Christensen $O B$ (2012) The spread amongst ensembles regional scenarios: regional climate models, driving general circulation models and interannual variability. Clim Dyn 38(5):951-964. doi:10.1007/s00382-011-1053-x

Dickinson RE, Errico RM, Giorgi F, Bates GT (1989) A regional climate model for the western United States. Clim Change 15:383-422. doi:10.1007/BF00240465

Done JM, Holland GJ, Bruyère CL, Leung LR, Suzuki-Parker A (2015) Modeling high-impact weather and climate: lessons from a tropical cyclone perspective. Clim Change 129:381-395. doi:10.1007/s10584-013-0954-6

Evans JP, Ji F, Lee C, Smith P, Argüeso D, Fita L (2014) Design of a regional climate modelling projection ensemble experiment-NARCliM. Geosci Model Dev 7:621-629. doi:10.5194/gmd-7-621-2014

Giorgi F, Bates GT (1989) The climatological skill of a regional model over complex terrain. Mon Wea Rev 117:2325-2347. http://doi.org/10.1175/ 1520-0493(1989)117\%3C2325:TCSOAR\%3E2.0.CO;2

Giorgi F, Shields C (1999) Tests of precipitation parameterizations available in latest version of NCAR Regional Climate Model (RegCM) over continental United States. J Geophys Res 104:6353-6375. doi:10.1029/98JD01164

Holland GJ, Done J, Bruyere C, Cooper C, Suzuki A (2010) Model investigations of the effects of climate variability and change on future Gulf of Mexico tropical cyclone activity. In: Proc. Offshore Technology Conf. Offshore Technology Conference, Texas. Offshore Technology Conference. doi:10.4043/20690-MS

Jin JM, Wang SY, Gillies RR (2011) An improved dynamical downscaling for the western United States. In: Blanco J (ed). Climate Change: Research and Technology for Adaptation and Mitigation. InTech, Rijeka Croatia. pp 23-38. Chap. 2. doi:10.5772/22991

Jones RG, Murphy JM, Noguer M (1995) Simulation of climate change over Europe using a nested regional-climate model. Part I: Assessment of control climate, including sensitivity to location of lateral boundaries. Quart J R Meteorol Soc 121:1413-1449. doi:10.1002/qj.49712152610

Kanada S, Nakano M, Kato T (2012) Projections of future changes in precipitation and the vertical structure of the frontal zone during the Baiu season in the vicinity of Japan using a 5-km-mesh regional climate model. J Meteorol Soc Japan 90A:65-86. doi:10.2151/jmsj.2012-A03

Kato H, Nishizawa K, Hirakuchi H, Kadokura S, Oshima N, Giorgi F (2001) Performance of RegCM2.5/NCAR-CSM nested system for the simulation of climate change in East Asia caused by global warming. J Meteorol Soc Japan 79:99-121. doi:10.2151/jmsj.79.99

Kawase H, Yoshikane T, Hara M, Ailikun B, Kimura F, Yasunari T (2008) Downscaling of the climatic change in the Mei-yu rainband in East Asia by a pseudo climate simulation method. SOLA 4. doi:10.2151/sola.2008-019

Kawase H, Yoshikane T, Hara M, Kimura F, Yasunari T, Ailikun B, Ueda H, Inoue T (2009) Intermodel variability of future changes in the Baiu rainband estimated by the pseudo global warming downscaling method. J Geophys Res 114(D24110). doi:10.1029/2009JD011803

Kimura F, Kitoh A (2007) Downscaling by pseudo global warming method. The final report of ICCAP. Research Institute for Humanity and Nature, Kyoto

Knutson TR, Sirutis JJ, Zhao M, Tuleya RE, Bender M, Vecchi GA, Villarini G, Chavas D (2015) Global projections of intense tropical cyclone activity for the late twenty-first century from dynamical downscaling of CMIP5/RCP4.5 scenarios. J Climate 28(18):7203-7224. doi:10.1175/JCLI-D-15-0129.1

Kröner N, Kotlarski S, Fischer E, Lüthi D, Zubler E, Schär C (2016) Separating climate change signals into thermodynamic, lapse-rate and circulation effects: theory and application to the European summer climate. Clim Dyn. doi:10.1007/s00382-016-3276-3

Kusaka H, Kondo H, Kikegawa Y, Kimura F (2001) A simple single-layer urban canopy model for atmospheric models: comparison with multi-layer and slab models. Bound-Layer Meteorol 101(3):329-358. doi:10.1023/A:1019207923078

Kusunoki S, Mizuta R, Matsueda M (2011) Future changes in the East Asian rain band projected by global atmospheric models with $20-\mathrm{km}$ and $60-\mathrm{km}$ grid size. Clim Dyn 37:2481-2493. doi:10.1007/s00382-011-1000-x

Mearns O, Giorgi F, McDaniel L, Shields C (1995) Analysis of daily variability of precipitation in a nested regional climate model: comparison with observations and doubled $\mathrm{CO} 2$ results. Global Planet Change 10:55-78. doi:10.1016/0921-8181(94)00020-E

Mearns L. O, Sain S, Leung LR, Bukovsky MS, McGinnis S, Biner S, Caya D, Arritt RW, Gutowski W, Takle E, Snyder M, Jones RG, Nunes AMB, Tucker S, Herzmann D, McDaniel L, Sloan L (2013) Climate change projections of the North American Regional Climate change Assessment Program (NARCCAP). Clim Change 120:965-975. doi:10.1007/s10584-013-0831-3

Misra V, Kanamitsu M (2004) Anomaly nesting: a methodology to downscale seasonal climate simulations from AGCMs. J Climate 17:3249-3262. http:// doi.org/10.1175/1520-0442(2004)017\%3C3249:ANAMTD\%3E2.0.CO;2

Mizuta R, Yoshimura H, Murakami H, Matsueda M, Endo H, Ose T, Kamiguchi K, Hosaka M, Sugi M, Yukimoto S, Kusunoki S, Kitoh A (2012) Climate simulations using MRI-AGCM3.2 with 20-km grid. J Meteorol Soc Japan 90A:233-258. doi:10.2151/jmsj.2012-A12

Mizuta R, Arakawa O, Ose T, Kusunoki S, Endo H, Kitoh A (2014) Classification of CMIP5 future climate responses by the tropical sea surface temperature changes. SOLA 10:167-171. doi:10.2151/sola.2014-035

Murakami H, Wang Y, Yoshimura H, Mizuta R, Sugi M, Shindo E, Adachi Y, Yukimoto S, Hosaka M, Kusunoki S, Ose T, Kitoh A (2012) Future changes in tropical cyclone activity projected by the new high-resolution MRI-AGCM. J Clim 25:3237-3260. doi:10.1175/JCLI-D-11-00415.1

Nakanishi M, Niino H (2009) Development of an improved turbulence closure model for the atmospheric boundary layer. J Meteorol Soc Japan 87:895-912. doi:10.2151/jmsj.87.895

Nishizawa S, Yashiro H, Sato Y, Miyamoto Y, Tomita H (2015) Influence of grid aspect ratio on planetary boundary layer turbulence in large-eddy simulations. Geosci Model Dev 8:3393-3419. doi:10.5194/gmd-8-33932015

Patricola CM, Cook KH (2010) Northern African climate at the end of the twenty-first century: an integrated application of regional and global climate models. Clim Dyn 35:193-212. doi:10.1007/s00382-009-0623-7

Rasmussen R, Liu C, Ikeda K, Gochis D, Yates D, Chen F, Tewari M, Barlage M, Dudhia J, Yu W, Miller K (2011) High-resolution coupled climate runoff simulations of seasonal snowfall over Colorado: a process study of current and warmer climate. J Clim 24:3015-3048. doi:10.1175/2010JCLI3985.1

Rayner NA, Parker DE, Horton EB, Folland CK, Alexander LV, Rowell DP, Kent EC, Kaplan A (2003) Global analyses of sea surface temperature, sea ice, and night marine air temperature since the late nineteenth century. J Geophys Res 108(D14). doi:10.1029/2002JD002670

Roberts MJ, Vidale PL, Mizielinski MS, Demory M-E, Schiemann R, Strachan J, Hodges K, Bell R, Camp J (2015) Tropical cyclones in the UPSCALE ensemble of high-resolution global climate models. J Climate 28(2):574-596. doi:10.1175/JCLI-D-14-00131.1

Rowell DP, Jones RG (2006) Causes and uncertainty of future summer drying over Europe. Clim Dyn 27:281-299. doi:10.1007/s00382-006-0125-9

Rummukainen M, Rockel B, Bärring L, Christensen JH, Reckermann M (2015) Twenty-first-century challenges in regional climate modeling. Bull Amer Meteorol Soc 96:135-138. doi:10.1175/BAMS-D-14-00214.1

Sato T, Kimura F, Kitoh A (2007) Projection of global warming onto regional precipitation over Mongolia using a regional climate model. J Hydrol 333:144-154. doi:10.1016/j.jhydrol.2006.07.023

Sato Y, Nishizawa S, Yashiro H, Miyamoto Y, Kajikawa Y, Tomita H (2015) Impacts of cloud microphysics on trade wind cumulus: which cloud microphysics processes contribute to the diversity in a large eddy simulation? Prog Earth Planet Sci 2. doi:10.1186/s40645-015-0053-6

Schär C, Frei C, Lüthi D, Davies HC (1996) Surrogate climate-change scenarios for regional climate models. Geophys Res Lett 23:669-672. doi:10.1029/96GL00265

Sekiguchi M, Nakajima T (2008) A $k$-distribution-based radiation code and its computational optimization for an atmospheric general circulation model. J Quant Spectrosc Radiat Transf 109:2779-2793. doi:10.1016/j.jqsrt.2008.07.013 
Takayabu I, Kanamaru H, Dairaku K, Benestad R, von Storch H, Christensen JH (2016) Reconsidering the quality and utility of downscaling. J Meteorol Soc Japan Ser II 94A:31-45. doi:10.2151/jmsj.2015-042

Taylor KE, Stouffer RJ, Meehl GA (2012) An overview of CMIP5 and the experiment design. Bull Am Meteorol Soc 93:485-498. doi:10.1175/BAMS-D-11-00094.1

Tomita H (2008) New microphysical schemes with five and six categories by diagnostic generation of cloud ice. J Meteorol Soc Japan 86A:121-142. doi:10.2151/jmsj.86A.121

van der Linden P, Mitchell FB (2009) ENSEMBLES: climate change and its impacts: summary of research and results from the ENSEMBLES project. technical report. Met Office Hadley Centre, FitzRoy Road, Exeter, EX1 3PB, U. K

van Oldenborgh GJ, Reyes FJD, Drijfhout SS, Hawkins E (2013) Reliability of regional climate model trends. Environ Res Lett 8. doi:10.1088/1748-9326/8/1/014055

Wakazuki Y, Rasmussen R (2015) Incremental dynamical downscaling for probabilistic analysis based on multiple GCM projections. Geophys Res Lett 42:10847-10855. doi:10.1002/2015GL066242

Walsh K, McGregor JL (1995) January and July climate simulations over the Australian region using a limited-area model. J Clim 8:2387-2403. http:// doi.org/10.1175/1520-0442(1995)008\%3C2387:JAJCSO\%3E2.0.CO;2

Wang Y, Leung LR, McGregor JL, Lee D-K, Wang W-C, Ding Y, Kimura F (2004) Regional climate modeling: Progress, challenges and prospects. J Meteorol Soc Japan 82:1599-1628. doi:10.2151/jmsj.82.1599

Wilson DK (2001) An alternative function for the wind and temperature gradients in unstable surface layers. Bound-Layer Meteorol 99:151-158. doi:10.1023/A:1018718707419

Wu W, Lynch AH (2000) Response of the seasonal carbon cycle in high latitudes to climate anomalies. J Geophys Res 105(D18):22897-22908. doi:10.1029/2000JD900340

Xu Z, Yang Z-L (2012) An improved dynamical downscaling method with GCM bias corrections and its validation with 30 years of climate simulations. J Clim 25:6271-6286. doi:10.1175/JCLI-D-12-00005.1

Ying M, Knutson TR, Kamahori H, Lee T-C (2012) Impacts of climate change on tropical cyclones in the western north pacific basin. part II: Late twenty-first century projections. Tropical Cyclone Res Rev 1:231-241

Yoshikane T, Kimura F, Kawase H, Nozawa T (2012) Verification of the performance of the pseudo-global-warming method for future climate changes during June in East Asia. SOLA 8:133-136. doi:10.2151/sola.2012-033

\section{Submit your manuscript to a SpringerOpen ${ }^{\circ}$ journal and benefit from:}

- Convenient online submission

- Rigorous peer review

- Open access: articles freely available online

- High visibility within the field

- Retaining the copyright to your article

Submit your next manuscript at $\gg$ springeropen.com 